\title{
ANOTHER PROOF OF WRIGHT'S INEQUALITIES
}

\author{
VLADY RAVELOMANANA
}

\begin{abstract}
We present a short way of proving the inequalities obtained by Wright in [Journal of Graph Theory, 4: 393 - 407 (1980)] concerning the number of connected graphs with $\ell$ edges more than vertices.
\end{abstract}

\section{Preliminaries}

For $n \geq 0$ and $-1 \leq \ell \leq\left(\begin{array}{l}n \\ 2\end{array}\right)-n$, let $c(n, n+\ell)$ be the number of connected graphs with $n$ vertices and $n+\ell$ edges. Quantifying $c(n, n+\ell)$ represents one of the fundamental tasks in the theory of random graphs. It has been extensively studied since the Erdös-Rényi's paper [3]. The generating functions associated to the numbers $c(n, n+\ell)$ are due to Sir E. M. Wright in a series of papers including [11, 12]. He also obtained the asymptotic formula for $c(n, n+\ell)$ for every $\ell=o\left(n^{1 / 3}\right)$. Using different methods, Bender, Canfield and McKay [1], Pittel and Wormald [8] and van der Hofstad and Spencer [9] were able to determine the asymptotic value of $c(n, n+\ell)$ for all ranges of $n$ and $\ell$.

For $\ell \geq-1$, let $W_{\ell}$ be the exponential generating function (EGF, for short) of the family of connected graphs with $n$ vertices and $n+\ell$ edges. Thus, $W_{\ell}(z)=\sum_{n=0}^{\infty} c(n, n+\ell) \frac{z^{n}}{n !}$. Let $T(z)$ be the EGF of the Cayley's rooted labeled trees. It is well known that $T(z)=$ $z e^{T(z)}=\sum_{n \geq 1} n^{n-1} \frac{z^{n}}{n !}$ (see for example 4 , 51). Among other results, Wright proved that the functions $W_{\ell}(z), \ell \geq-1$, can be expressed in terms of $T(z)$. Such results allowed penetrating and precise analysis when studying random graphs processes as it has been shown for example in the giant paper [5]. Throughout the rest of this note, all formal power series are univariate. Therefore, for sake of simplicity we will often omit the variable $z$ so that $T \equiv T(z), W_{i} \equiv W_{i}(z)$ and so on.

We need the following notations.

Definition. If $A$ and $B$ are two formal power series such that for all $n \geq 0$ we have $\left[z^{n}\right] A(z) \leq\left[z^{n}\right] B(z)$ then we denote this relation $A \preceq B$ or $A(z) \preceq B(z)$.

The aim of this note is to provide an alternative and generating function based proof of the inequalities obtained by Sir Wright in [12] (in particular, he used numerous intermediate lemmas). More precisely, Wright obtained the following. 
Theorem (Wright 1980). Let $b_{1}=\frac{5}{24}$ and $c_{1}=\frac{19}{24}$. Define recursively $b_{\ell}$ and $c_{\ell}$ by

$$
2(\ell+1) b_{\ell+1}=3 \ell(\ell+1) b_{\ell}+3 \sum_{t=1}^{\ell-1} t(\ell-t) b_{t} b_{\ell-t}, \quad(\ell \geq 1)
$$

and

$$
\begin{aligned}
2(3 \ell+2) c_{\ell+1} & =8(\ell+1) b_{\ell+1}+3 \ell b_{\ell}+(3 \ell+2)(3 \ell-1) c_{\ell} \\
& +6 \sum_{t=1}^{\ell-1} t(3 \ell-3 t-1) b_{t} c_{\ell-t}, \quad(\ell \geq 1)
\end{aligned}
$$

Then, for all $\ell \geq 1$

$$
\frac{b_{\ell}}{(1-T(z))^{3 \ell}}-\frac{c_{\ell}}{(1-T(z))^{3 \ell-1}} \preceq W_{\ell}(z) \preceq \frac{b_{\ell}}{(1-T(z))^{3 \ell}} \quad .
$$

(3) is known as Wright's inequalities and such results has been extremely useful in the enumerative study of graphs as well as in the theory of random graphs [2, 5, 6, 7, 10].

Our proof of (3) is based upon two ingredients:

Fact 1 . We know that the EGFs $W_{\ell}$ satisfy $W_{-1}=T-\frac{T^{2}}{2}, W_{0}=-\frac{1}{2} \log (1-T)-\frac{T}{2}-\frac{T^{2}}{4}$ and

$$
(1-T) \vartheta_{z} W_{\ell+1}+(\ell+1) W_{\ell+1}=\left(\frac{\vartheta_{z}^{2}-3 \vartheta_{z}}{2}-\ell\right) W_{\ell}+\frac{1}{2} \sum_{k=0}^{\ell}\left(\vartheta_{z} W_{k}\right)\left(\vartheta_{z} W_{\ell-k}\right), \quad(\ell \geq 0)
$$

where $T=T(z), W_{k}=W_{k}(z)$ and $\vartheta_{z}=z \frac{\partial}{\partial z}$ corresponds to marking a vertex (such combinatorial operator consists to choose a vertex among the others). For the combinatorial sense of (田), we refer the reader to [1, 5] or [11].

Fact 2. Let $A$ and $B$ be two formal power series and $\ell \in \mathbb{N}$. If $(1-T) \vartheta_{z} A+(\ell+1) A \preceq$ $(1-T) \vartheta_{z} B+(\ell+1) B$ then $A \preceq B$.

To prove Fact 2 , fix $\ell \geq 0$. We write

$$
B(z)-A(z)=\sum_{n=0}^{\infty}\left(b_{n}-a_{n}\right) \frac{z^{n}}{n !} \quad \text { and } \quad \forall n, c_{n}=b_{n}-a_{n} .
$$

Suppose that $(1-T) \vartheta_{z} A+(\ell+1) A \preceq(1-T) \vartheta_{z} B+(\ell+1) B$. We then have

$$
\begin{gathered}
n !\left[z^{n}\right]\left((1-T(z)) \vartheta_{z}(B(z)-A(z))+(\ell+1)(B(z)-A(z))\right)= \\
(n+\ell+1) c_{n}-\sum_{k=1}^{n}\left(\begin{array}{l}
n \\
k
\end{array}\right) k^{k-1}(n-k) c_{n-k} \geq 0 .
\end{gathered}
$$

It is now easily seen that $\forall n, c_{n} \geq 0$. Therefore, $A \preceq B$.

Our proof of (3) is divided into two parts each of each are given in the next Sections. 


\section{PROOF OF $W_{\ell} \preceq \frac{b_{\ell}}{(1-T)^{3 \ell}}$}

Define the family $\left(\bar{W}_{\ell}\right)_{\ell \geq 0}$ as $\bar{W}_{0}=-\frac{1}{2} \log (1-T)$ and for $\ell \in \mathbb{N}^{\star}, \bar{W}_{\ell}=\frac{b_{\ell}}{(1-T)^{3 \ell}}$. Observe that we have $W_{0} \preceq \bar{W}_{0}$ and $W_{1} \preceq \bar{W}_{1}$ has been proved in [12]. Now, we can proceed by induction. Suppose that for $2 \leq i \leq \ell, W_{i} \preceq \bar{W}_{i}=\frac{b_{i}}{(1-T)^{3 i}}$ and let us prove that $W_{\ell+1} \preceq \bar{W}_{\ell+1}=\frac{b_{\ell+1}}{(1-T)^{3 \ell+3}}$. Simple calculations show that

$$
\left(\frac{\vartheta_{z}^{2}-\vartheta_{z}}{2}\right) \bar{W}_{\ell} \preceq \frac{\vartheta_{z}^{2}}{2} \bar{W}_{\ell} \preceq \frac{3 \ell(3 \ell+2)}{2} \frac{b_{\ell}}{(1-T)^{3 \ell+4}}-\frac{3 \ell(3 \ell+2)}{2} \frac{b_{\ell}}{(1-T)^{3 \ell+3}},
$$

$$
\left(\vartheta_{z} \bar{W}_{0}\right) \quad\left(\vartheta_{z} \bar{W}_{\ell}\right) \preceq \frac{3 \ell b_{\ell}}{2} \frac{b_{\ell}}{(1-T)^{3 \ell+4}}-\frac{3 \ell b_{\ell}}{2} \frac{b_{\ell}}{(1-T)^{3 \ell+3}} \quad \text { and }
$$

(9) $\frac{1}{2} \sum_{p=1}^{\ell-1}\left(\vartheta_{z} \bar{W}_{p}\right)\left(\vartheta_{z} \bar{W}_{\ell-p}\right) \preceq \frac{1}{2}\left(\sum_{p=1}^{\ell-1} 9 p(\ell-p) b_{p} b_{\ell-p}\right)\left(\frac{1}{(1-T)^{3 \ell+4}}-\frac{1}{(1-T)^{3 \ell+3}}\right)$.

Summing (7), (8), (9), using the recurrence (11) and the induction hypothesis, we find that

$$
(1-T) \vartheta_{z} W_{\ell+1}+(\ell+1) W_{\ell+1} \preceq \frac{3(\ell+1) b_{\ell+1}}{(1-T)^{3 \ell+4}}-\frac{3(\ell+1) b_{\ell+1}}{(1-T)^{3 \ell+3}} .
$$

Since

$$
(1-T) \vartheta_{z} \bar{W}_{\ell+1}+(\ell+1) \bar{W}_{\ell+1}=\frac{3(\ell+1) b_{\ell+1}}{(1-T)^{3 \ell+4}}-\frac{2(\ell+1) b_{\ell+1}}{(1-T)^{3 \ell+3}}
$$

by Fact 2 , we have $\bar{W}_{\ell+1} \succeq W_{\ell+1}$.

\section{Proof OF $\frac{b_{\ell}}{(1-T)^{3 \ell}}-\frac{c_{\ell}}{(1-T)^{3 \ell-1}} \preceq W_{\ell}$}

Define $\underline{W}_{0}=W_{0}$ and for $\ell \in \mathbb{N}^{\star}, \underline{W}_{\ell}=\frac{b_{\ell}}{(1-T)^{3 \ell}}-\frac{c_{\ell}}{(1-T)^{3 \ell-1}}$. As before, we shall proceed by induction. We have $\underline{W}_{0} \preceq W_{0}$ and

$$
W_{1}-\underline{W}_{1}=\frac{13}{12(1-T)}-\frac{1}{2}-\frac{T}{8}+\frac{T^{2}}{24} \succeq \frac{13}{12}\left(\frac{1}{(1-T)}-T-1\right)=\frac{13 T^{2}}{12(1-T)} \succeq 0 .
$$

Suppose that for $2 \leq k \leq \ell, \underline{W}_{k}=\frac{b_{k}}{(1-T)^{3 k}}-\frac{c_{k}}{(1-T)^{3 k-1}} \preceq W_{k}$. We have to prove that $\underline{W}_{\ell+1}=\frac{b_{\ell+1}}{(1-T)^{3 \ell+3}}-\frac{c_{\ell+1}}{(1-T)^{3 \ell+2}} \preceq W_{\ell+1}$. For this purpose, define $\Psi_{\ell+1}$ as

$$
\begin{aligned}
\Psi_{\ell+1} & =\left(\frac{\vartheta_{z}^{2}-3 \vartheta_{z}}{2}-\ell\right) \underline{W}_{\ell}+\left(\vartheta_{z} \underline{W}_{0}\right)\left(\vartheta_{z} \underline{W}_{\ell}\right)+\frac{1}{2} \sum_{k=1}^{\ell-1}\left(\vartheta_{z} \underline{W}_{k}-\frac{(3 \ell-1) c_{\ell}}{(1-T)^{3 \ell}}\right)\left(\vartheta_{z} \underline{W}_{\ell-k}\right) \\
& -\left(\frac{\alpha_{\ell}}{(1-T)^{3 \ell+2}}+\frac{\beta_{\ell}}{(1-T)^{3 \ell+1}}+\frac{\gamma_{\ell}}{(1-T)^{3 \ell}}+\frac{\delta_{\ell}}{(1-T)^{3 \ell-1}}\right),
\end{aligned}
$$


where $\alpha_{\ell}, \beta_{\ell}, \gamma_{\ell}$ and $\delta_{\ell}$ are given by

$$
\begin{aligned}
\alpha_{\ell} & =\frac{(7 \ell+4) c_{\ell+1}}{2}-3(\ell+1) b_{\ell+1}-\frac{3}{4} \ell b_{\ell}+\frac{(3 \ell-1)(3 \ell+4)}{4} c_{\ell} \\
& +\frac{1}{2} \sum_{t=1}^{\ell-1}(3 t-1) c_{t}(3 \ell-3 t-1) c_{\ell-t}, \\
\beta_{\ell} & =-\frac{(3 \ell+2) c_{\ell+1}}{2}+2(\ell+1) b_{\ell+1}-\frac{3}{4} \ell b_{\ell}-\frac{(3 \ell-1)(3 \ell+4)}{4} c_{\ell} \\
& -\frac{1}{2} \sum_{t=1}^{\ell-1}(3 t-1) c_{t}(3 \ell-3 t-1) c_{\ell-t}, \\
& \gamma_{\ell}=\frac{\ell b_{\ell}}{2}+\frac{(3 \ell-1) c_{\ell}}{2} \text { and } \delta_{\ell}=-\frac{\ell-1}{2} c_{\ell} .
\end{aligned}
$$

Rewritting the formal power series $\frac{\alpha_{\ell}}{(1-T)^{3 \ell+2}}+\frac{\beta_{\ell}}{(1-T)^{3 \ell+1}}+\frac{\gamma_{\ell}}{(1-T)^{3 \ell}}+\frac{\delta_{\ell}}{(1-T)^{3 \ell-1}}$ as follows

$$
\begin{aligned}
& \frac{(7 \ell+4) / 2 c_{\ell+1}-3(\ell+1) b_{\ell+1}-3 / 4 \ell b_{\ell}}{(1-T)^{3 \ell+2}}-\frac{(3 \ell+2) / 2 c_{\ell+1}-2(\ell+1) b_{\ell+1}+3 / 4 \ell b_{\ell}}{(1-T)^{3 \ell+1}} \\
& +(3 \ell-1)(3 \ell+4) c_{\ell}\left(\frac{1}{(1-T)^{3 \ell+2}}-\frac{1}{(1-T)^{3 \ell+1}}\right) \\
& +\frac{2 \ell b_{\ell}}{2(1-T)^{3 \ell}}+\left(\frac{(3 \ell-1) c_{\ell}}{2(1-T)^{3 \ell}}-\frac{(\ell-1) c_{\ell}}{2(1-T)^{3 \ell-1}}\right)
\end{aligned}
$$

it is easily seen that if the quantity (coming from the denominators of the 2 first terms of the above equation)

$$
(2 \ell+1) c_{\ell+1}-(\ell+1) b_{\ell+1}-\frac{3}{2} \ell b_{\ell} \geq 0
$$

then $\frac{\alpha_{\ell}}{(1-T)^{3 \ell+2}}+\frac{\beta_{\ell}}{(1-T)^{3 \ell+1}}+\frac{\gamma_{\ell}}{(1-T)^{3 \ell}}+\frac{\delta_{\ell}}{(1-T)^{3 \ell-1}} \succeq 0$. (We used $1 /(1-T)^{a} \succeq 1 /(1-T)^{b}$ if $a \geq b)$.

Using (11) and (2), after simple algebra we have (18). Therefore by construction, RHS of(4) $\Psi_{\ell+1}$. After nice cancellations, it yields

$$
\Psi_{\ell+1}=\frac{3(\ell+1) b_{\ell+1}}{(1-T)^{3 \ell+4}}-\frac{2(\ell+1) b_{\ell+1}+(3 \ell+2) c_{\ell+1}}{(1-T)^{3 \ell+3}}+\frac{(2 \ell+1) c_{\ell+1}}{(1-T)^{3 \ell+2}} .
$$

Remarking that $(1-T) \vartheta_{z} \underline{W}_{\ell+1}+(\ell+1) \underline{W}_{\ell+1}=\Psi_{\ell+1}$, we have completed the proof of $\underline{W}_{\ell+1} \preceq W_{\ell+1}$.

\section{REFERENCES}

[1] Bender, E. A., Canfield, E. R. and McKay B. D. (1990). The asymptotic number of labelled connected graphs with a given number of vertices and edges. Random Structures and Algorithms, 1:127-169.

[2] Bollobás, B. (1985). Random Graphs. Academic Press, London.

[3] Erdős, P. and Rényi A. (1959). On random graphs. Publ. Math. Debrecen, 6:290-297.

[4] Flajolet, P. and Sedgewick, R. Analytic Combinatorics. To appear (chapters are avalaible as Inria research reports). See http://algo.inria.fr/flajolet/Publications/books.html. 
[5] Janson, S., Knuth, D. E., Luczak, T. and Pittel B. (1993). The birth of the giant component. Random Structures and Algorithms, 4:233-358.

[6] Janson, S., Łuczak, T. and Ruciński A. (2000). Random Graphs. John Wiley, New York.

[7] Euczak, T. (1990). On the number of sparse connected graphs. Random Structures and Algorithms, $1: 171-174$

[8] Pittel, B. and Wormald, N. C. (2005). Counting connected graphs inside out. J. Combinatorial Th. Ser. $B, 93: 127-172$.

[9] van der Hofstad, R. and Spencer. J. (2006). Counting Connected Graphs Asymptotically. European Journal on Combinatorics, 27: 1294-1320.

[10] Ravelomanana (2006). The Average Size of Giant Components between the Double-Jump. Algorithmica, 46: 529-555.

[11] Wright, E. M. (1977). The Number of Connected Sparsely Edged Graphs. Journal of Graph Theory, $1: 317-330$

[12] Wright, E. M. (1980). The Number of Connected Sparsely Edged Graphs III: Asymptotic results. Journal of Graph Theory, 4:393-407.

E-mail address: vlad@lipn.univ-paris13.fr

Vlady RAVELOMANANA, LIPN - UMR 7030, Institut Galilée -Université de Paris-Nord, 99, Avenue J. B. Clément. F 93430 Villetaneuse, France. 